\title{
CORRECTION
}

\section{Correction to: Surgical correction of double major adolescent idiopathic scoliosis}

\author{
Cesare Faldini ${ }^{1}$ - Alberto Ruffilli ${ }^{1} \cdot$ Fabrizio Perna $^{1} \cdot$ Federico Pilla $^{1}$ - Alessandro Panciera ${ }^{1} \cdot$ Francesco Traina $^{1}$
}

Published online: 31 October 2018

(c) Springer-Verlag GmbH Germany, part of Springer Nature 2018

\section{Correction: \\ European Spine Journal (2018) 27 (Suppl 4):S571-S573 \\ https://doi.org/10.1007/s00586-018-5662-5}

Unfortunately, the affiliation of the author group has been incorrectly published in original version. The complete correct affiliation of all authors should read as follows.

IRCCS Istituto Ortopedico Rizzoli, Via Giulio Cesare Pupilli 1, 40136, Bologna, Italy

The original article can be found online at https://doi.org/10.1007/ s00586-018-5662-5.

Cesare Faldini

cesare.faldini@ior.it

1 IRCCS Istituto Ortopedico Rizzoli, Via Giulio Cesare Pupilli

1, 40136 Bologna, Italy 